\title{
Carbohydrate-Deficient Transferrin: Validity of a New Alcohol Biomarker in a Sample of Patients with Diabetes and Hypertension
}

\author{
Michael Fleming, MD, MPH, and Marlon Mundt, MS
}

Objective: To test the reliability and validity of a new alcohol biomarker.

Design: Cross-sectional with matched control. Alcohol use, symptoms of alcohol dependence, health status, current medications, carbohydrate-deficient transferrin (CDT) levels, and $\gamma$-glutamyl transferase levels were assessed. Validity of the \%CDT test was estimated using a drinking cutoff of 90 or more drinks in the previous 30 days.

Participants: Sample $(n=799)$ included 89 patients with type 2 diabetes, 299 patients with hypertension, 209 patients with hypertension and type 2 diabetes, and 202 matched control subjects with neither diabetes nor hypertension.

Results: Three hundred ninety-six women and 403 men ages 30 to 60 participated in the study. Six percent ( 45 of 799$)$ reported drinking more than 14 drinks per week, $2 \%(n=17)$ met criteria for alcohol abuse, and 3\% (n=23) met DSM-IV criteria for dependence. The overall sensitivity of the $\%$ CDT test was $61 \%$ with a specificity of $85 \%$. A receiver operating characteristic curve was used to determine that the best fit was for persons who reported $>90$ drinks per month and a CDT level $>2.5 \%$ with an area under the curve of 0.83 . Test-retest reliability $(R)$ was 0.94 .

Conclusions: \%CDT levels are useful in detecting and/or confirming high-risk drinking in patients being treated for type 2 diabetes and hypertension. (J Am Board Fam Pract 2004;17:247-55.)

Alcohol use is an important factor in the etiology and treatment of type 2 diabetes and hypertension. Alcohol use can affect glucose levels and blood pressure $^{1-4}$ and increase the risk for medical complications, such as ketoacidosis, peripheral vascular disease, peripheral neuropathy, heart disease, and cerebrovascular accidents. ${ }^{5-8}$ Although there is a continuing debate on how much is too much for persons with diabetes and hypertension, ${ }^{9}$ consistent evidence shows that high doses are harmful. ${ }^{10}$

As a result, there has been increasing interest in developing more accurate alcohol screening questions and laboratory tests to identify and treat patients who use alcohol above recommended limits. The current standard of care includes asking consumption questions, administering the CAGE

Submitted, revised, 8 December 2003.

From the Department of Family Medicine, University of Wisconsin Medical School, Madison. Address correspondence to Michael Fleming, MD, 777 South Mills Street, Madison, WI 53715 (E-mail: mfleming@fammed.wisc.edu).

This work was supported by Axis-Shield of Oslo Norway, The National Institute on Alcohol Abuse and Alcoholism, the University of Wisconsin Department of Family Medicine Research Program, the University of Wisconsin Hospital and Clinics Laboratories, and the Group Health Cooperative of Wisconsin. questions, or using a screening questionnaire such as the Alcohol Use Disorder Inventory Test. ${ }^{11}$ Clinical experience, however, suggests that patients often minimize their alcohol use, especially those who are alcohol-dependent. Simply asking patients how much they drink is likely to miss a significant number of persons who could benefit from a reduction in alcohol use.

Traditional alcohol biomarkers include blood alcohol levels, liver function tests, such as $\gamma$-glutamyl transferase (GGT), and hematologic tests, such as mean corpuscular hemoglobin (MCV). Another method involves combining a number of routine blood chemistries, high-density lipoprotein levels, and hematologic measures using discriminant function analysis techniques. ${ }^{12}$ Alcohol biomarkers currently being tested in research studies include hemoglobin-associated acetaldehyde, fatty acid esters, and $\beta$-hexosaminidase isoenzyme $\mathrm{B}$ activity. ${ }^{13,14}$ None of these laboratory tests, however, is sufficiently sensitive or specific for screening or monitoring, and none of these tests has been approved by the FDA as an alcohol biomarker.

The first test to receive FDA approval ${ }^{15}$ as an alcohol biomarker was the carbohydrate-deficient transferrin (CDT) test. CDT was approved for use 
in the US health care system in 2001. This test has been used by life insurance companies in the US since 1995 to screen persons for heavy alcohol use. ${ }^{16}$ CDT is also widely used in Europe to monitor abstinence, assess preoperative alcohol risk, and identify high-risk drinking in surgical and medical settings. ${ }^{17-21}$

The goal of the study was to determine the reliability and validity of \%CDT levels in a sample of primary care patients being treated with medication for type 2 diabetes and/or hypertension using well-validated patient self-report as the criterion standard. This is the first large-scale study of CDT in general medical care settings in the United States.

\section{Methods \\ Design}

\%CDT levels were measured in a sample of 799 patients selected from 8 primary care clinics located in a rural/urban county in the Midwest. The CDT test used for this study was the \%CDT turbidimetric immunoassay (TIA) (Bio-Rad, Hercules, CA). To assess test/retest reliability, CDT levels were repeated at 2- and 4-months after baseline in persons who had an initial CDT test greater than $2.5 \%$ and in those subjects who reported drinking $>2$ drinks per day. Medical record reviews were conducted to determine physician identification of treatment of persons with elevated CDT levels. The University of Wisconsin Health Sciences Committee for the Protection of Human Subjects approved the study. Patient self-reported alcohol use, using a 30-day timeline follow-back interview, was used to determine the validity of the \%CDT test in this population.

\section{Sample Recruitment}

Subjects were recruited from 8 primary care sites. Four of the clinics were academic teaching sites of the University of Wisconsin Department of Family Medicine. The fifth site was a private practice site staffed by 7 primary care physicians. The 3 other primary care sites were part of a staff model HMO staffed by 21 primary care physicians. The sample was obtained with the assistance of 72 primary care physicians practicing in these 8 clinics.

Subjects between the ages of 30 and 60 were recruited into 1 of 4 groups. Group 1 consisted of patients being treated with medication for type 2 diabetes. Group 2 patients were being treated for hypertension. Group 3 subjects were being treated for both hypertension and diabetes. Group 4, the control group, consisted of patients who had neither diabetes nor hypertension. Some of the control subjects had other medical conditions, such as elevated lipids or heart disease.

Potential subjects were identified in the 8 primary care clinics through the use of ICD-9 billing codes using a clinic data warehouse. Computerized searches of the data warehouse used ICD-9 codes related to diabetes, hypertension, and their complications, such as renal, eye, and heart disease. A random selection process was used to select subjects for the study. Each selected subject received a letter from his/her physician with an invitation to participate in the study. An opt-out postcard method (subjects who did not return the postcard were contacted by telephone) was used in 5 clinics, and an opt-in procedure (subjects had to return a postcard before telephone contact) was used in 3 clinics.

Potential research subjects were then contacted by telephone, screened for eligibility criteria, and invited for a face-to-face interview and a blood test. Eligibility criteria included: currently taking medication for type 2 diabetes and/or hypertension, age 30 to 60 , and no current pregnancy. Persons with type 1 diabetes were not eligible for the study. Control group patients were obtained from the same clinical sites matched on age and gender. Signed informed consent was obtained at the time of the interview. Subjects were paid $\$ 50$ for the interview and blood test. Subjects who participated in the 2- and 4-month follow-up procedures were paid an additional $\$ 60$.

\section{Measurement of \%CDT}

Carbohydrate-deficient transferrin is present at low levels in most adults $(<2.6 \%$ of total transferrin levels), including persons who do not drink alcohol. Human transferrin (a carrier protein for iron) occurs in at least 6 isoforms. These isoforms include penta-, tetra-, tri-, di-, mono-, and asialo transferrin. The asialo, monosialo, and disialo isoforms of transferrin are referred to as carbohydratedeficient transferrin (CDT). The CDT isoforms of transferrin are elevated in persons who use excessive quantities of alcohol. There are also factors besides alcohol use that may affect CDT levels, including age, ${ }^{22}$ genetic variants, ${ }^{14}$ medication, ${ }^{23}$ 
female hormones, ${ }^{24}$ iron stores, ${ }^{25}$ tobacco use, ${ }^{26}$ and other factors. ${ }^{27,28}$

The \%CDT TIA is the test used for this study. Of the 12 tests that measure CDT levels, this is the only CDT test currently approved by the FDA in the US. The \%CDT TIA procedure measures the sum of CDT isoforms relative to the total amount of transferrin. The \%CDT TIA is a heterogeneous immunoassay with column separation followed by turbidimetric measurement. Serum transferrin in the sample is saturated with $\mathrm{Fe}^{3+}$. The mixture is applied to an ion-exchange column. Because of the different amounts of sialic residues on transferrin, the isoforms carry different charges and are separated in the column. The CDT isoforms are eluted. The CDT content of the collected eluate is determined by turbidimetric measurement. The eluted CDT isoforms form immune complexes with antitransferrin antibodies. Total transferrin content of the sample is determined separately, using the same anti-transferrin antibodies. The measurements are evaluated using a calibration curve, and the \%CDT value is calculated.

A research scientist at the University of Wisconsin Hospital Laboratories performed the \%CDT tests reported in the study. Validation of the \%CDT test was performed at 2 reference labs using methods developed by Bean. ${ }^{29}$

\section{Analysis}

Descriptive statistics were used to characterize the 4 groups who participated in the study. Alcohol use was determined using a 30-day calendar interview method. ${ }^{30} \mathrm{~A}$ standard drink was defined as 12 ounces of beer, 5 ounces of wine, 1.5 ounces of spirits, or $14 \mathrm{~g}$ of alcohol. The total amount of alcohol reported each day was added together to compute the total 30-day quantity. Drinkers were categorized as abstinent, 30 or more drinks in the previous month, 60 or more, 90 or more, and 120 or more. These categories correspond to 1, 2, 3, and 4 drinks per day, which are commonly used in epidemiologic studies. ${ }^{31}$

High-risk drinkers are defined as persons drinking 3 or more drinks per day and those who meet DSM-IV criteria for abuse or dependence. To meet criteria for current alcohol abuse subjects needed to be positive for at least 1 of the 3 DSM-IV abuse criteria. To meet criteria for dependence, subjects need to report a minimum of 3 of the 7 DSM-IV dependence criteria. The sensitivity and
\% CDT Interquartile Range by 30 Day Drinking

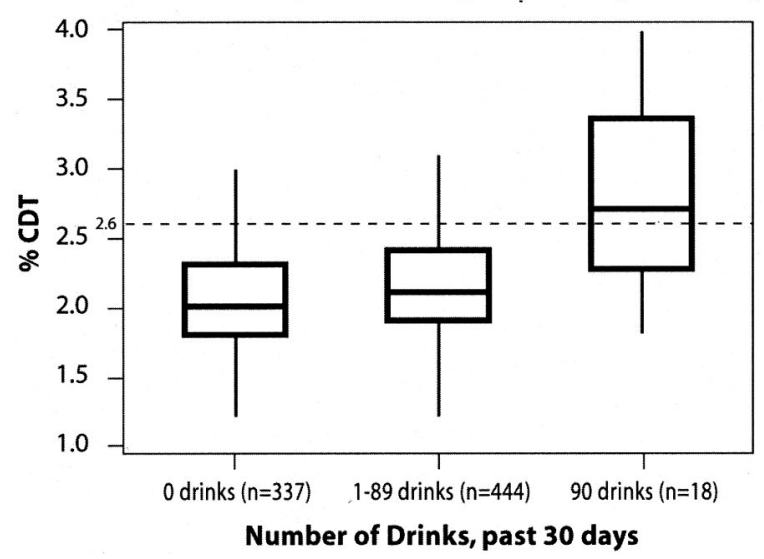

Figure 1. This figure displays the interquartile range of \%CDT values for subjects who were abstinent (0 drinks), moderate alcohol drinkers (1 to 89 drinks), and high-risk drinkers (90 or more drinks) in the past 30 days. The median \%CDT level increases from 2.0 for abstainers, to 2.1 for moderate drinkers, and to 2.7 for heavy drinkers. A \%CDT level of 2.6 or higher is used to positively identify heavy alcohol use.

specificity of the \%CDT test in the sample was calculated using 90 or more drinks in the previous 30 days as a true case and \%CDT $>2.5$ as a positive $\%$ CDT test. The box and whiskers plots of \% CDT scores reported in Figure 1 provide \%CDT interquartile ranges and medians by reported alcohol consumption. The receiver operating characteristic (ROC) curve plots sensitivity vs. ( 1 - specificity) of various cutoffs for positive \%CDT test results.

\section{Results}

A computer search of the data warehouse for the 8 clinics generated a list of 3016 patients with diabetes and/or hypertension. An additional list of 335 patients was generated for the control group matched on gender and age. Potential subjects were randomly selected to receive letters of invitation in batches of 30 to 50 letters until our sample of 799 subjects was obtained.

Of the 1608 patients mailed letters using the opt-out postcard method, 246 returned the opt-out postcard and declined to participate. Seven hundred twenty-four persons in this group met the inclusion criteria and were enrolled in the study. Of the 348 subjects mailed letters using the opt-in method, 71 subjects participated in the study. Subject recruitment was lower using the opt-in post- 
Table 1. Sociodemographics and Health Status of 799 Persons, 30 to 60 Years 0ld, Recruited from 8 Primary Care Clinics

\begin{tabular}{|c|c|c|c|c|c|}
\hline & $\begin{array}{l}\text { Diabetic Only } \\
\quad(\mathrm{n}=89)\end{array}$ & $\begin{array}{l}\text { Hypertensive Only } \\
\qquad(\mathrm{n}=299)\end{array}$ & $\begin{array}{c}\text { Both Diabetic } \\
\text { and Hypertensive } \\
(\mathrm{n}=209)\end{array}$ & $\begin{array}{l}\text { Neither Diabetic } \\
\text { nor Hypertensive } \\
\quad(\mathrm{n}=202)\end{array}$ & $\begin{array}{l}\text { All Patients } \\
(\mathrm{n}=799)\end{array}$ \\
\hline \multicolumn{6}{|l|}{ Gender } \\
\hline Female & $50(56 \%)$ & $146(49 \%)$ & $102(49 \%)$ & $98(49 \%)$ & $396(50 \%)$ \\
\hline Male & $39(44 \%)$ & $153(51 \%)$ & $107(51 \%)$ & $104(51 \%)$ & $403(50 \%)$ \\
\hline Age, mean & 48.5 & 49.8 & 51.7 & 47.9 & 49.7 \\
\hline \multicolumn{6}{|l|}{ Ethnicity } \\
\hline White & $71(80 \%)$ & $267(89 \%)$ & $177(85 \%)$ & $185(92 \%)$ & $700(88 \%)$ \\
\hline African American & $10(11 \%)$ & $21(7 \%)$ & $21(10 \%)$ & $13(6 \%)$ & $65(8 \%)$ \\
\hline Asian & $4(4 \%)$ & $5(2 \%)$ & $0(0 \%)$ & $2(1 \%)$ & $11(1 \%)$ \\
\hline Hispanic & $3(3 \%)$ & $5(2 \%)$ & $7(3 \%)$ & $2(1 \%)$ & $17(2 \%)$ \\
\hline Other & $1(1 \%)$ & $1(0 \%)$ & $4(2 \%)$ & $0(0 \%)$ & $6(1 \%)$ \\
\hline \multicolumn{6}{|l|}{ Education } \\
\hline High school or less & $33(37 \%)$ & $89(30 \%)$ & $68(33 \%)$ & $53(26 \%)$ & $243(30 \%)$ \\
\hline Technical school & $22(25 \%)$ & $56(19 \%)$ & $44(21 \%)$ & $38(19 \%)$ & $160(20 \%)$ \\
\hline College & $18(20 \%)$ & $97(32 \%)$ & $58(28 \%)$ & $57(28 \%)$ & $230(29 \%)$ \\
\hline Postgraduate & $16(18 \%)$ & $57(19 \%)$ & $38(18 \%)$ & $54(27 \%)$ & $165(21 \%)$ \\
\hline \multicolumn{6}{|l|}{ Household size } \\
\hline 1 & $13(15 \%)$ & $43(14 \%)$ & $29(14 \%)$ & $23(11 \%)$ & $108(14 \%)$ \\
\hline 2 & $37(42 \%)$ & $125(42 \%)$ & $107(51 \%)$ & $85(42 \%)$ & $354(44 \%)$ \\
\hline 3 to 4 & $34(38 \%)$ & $106(35 \%)$ & 47 (22\%) & $75(37 \%)$ & $262(33 \%)$ \\
\hline 5 or more & $5(6 \%)$ & $24(8 \%)$ & $24(11 \%)$ & $19(9 \%)$ & $72(9 \%)$ \\
\hline \multicolumn{6}{|l|}{ Marital status } \\
\hline Married/with partner & $60(68 \%)$ & $206(69 \%)$ & $136(65 \%)$ & $143(71 \%)$ & $545(68 \%)$ \\
\hline Never married & $12(13 \%)$ & $35(12 \%)$ & $21(10 \%)$ & $21(10 \%)$ & $89(11 \%)$ \\
\hline Separated/divorced & $13(15 \%)$ & $51(17 \%)$ & $44(21 \%)$ & $36(18 \%)$ & $144(18 \%)$ \\
\hline Widowed & $4(4 \%)$ & $7(2 \%)$ & $7(3 \%)$ & $2(1 \%)$ & $20(3 \%)$ \\
\hline \multicolumn{6}{|l|}{ Health problems* } \\
\hline Hepatitis C & $0(0 \%)$ & $2(1 \%)$ & $5(2 \%)$ & $4(2 \%)$ & $11(1 \%)$ \\
\hline Asthma & $13(15 \%)$ & $23(8 \%)$ & $37(18 \%)$ & $15(7 \%)$ & $88(11 \%)$ \\
\hline Depression & $27(30 \%)$ & $79(26 \%)$ & $65(31 \%)$ & $42(21 \%)$ & $213(27 \%)$ \\
\hline Anxiety & $31(35 \%)$ & $83(30 \%)$ & $66(32 \%)$ & $53(26 \%)$ & $233(29 \%)$ \\
\hline Elevated cholesterol & $39(44 \%)$ & $94(11 \%)$ & $117(56 \%)$ & $43(21 \%)$ & $293(37 \%)$ \\
\hline
\end{tabular}

* Categories are not mutually exclusive.

card method compared with the opt-out method ( $20 \%$ vs. $45 \%)$. Fifty-four subjects met 1 of the exclusion criteria and were not eligible for the study. Of the 799 patients who completed the baseline interview, 151 patients either had a baseline CDT level of $>2.5 \%$ or reported heavy alcohol use and participated in a face-to-face interview and blood draw at 2 and 4 months after baseline.

The sample included 396 women with a mean age of 50.1 and 403 men with a mean age of 49.4. Table 1 lists a number of characteristics of the sample. Sixty-eight percent were married or living with a partner. Eight percent were African American, $2 \%$ were Asian, $3 \%$ were Hispanic, and $87 \%$ were white. The mean education level was high, with $70 \%$ having more than a high school education. The majority of patients in the 4 groups reported at least 1 health problem (eg, asthma, depression, hepatitis C, or hyperlipidemia).
Rates of abstinence, alcohol use, abuse, and dependence among the 4 groups are illustrated in Table 2 . Nearly $42 \%(n=337)$ of the sample was abstinent; the highest rates of abstinence were in the diabetic sample. Six percent $(n=45)$ of subjects drank 2 or more drinks ( $28 \mathrm{~g}$ ) of alcohol per day and $2 \%(\mathrm{n}=18)$ reported drinking 3 or more drinks per day. Two percent met current criteria for alcohol abuse, and 3\% met criteria for dependence. Of the persons who met criteria for abuse or dependence, $10 \%$ reported abstinence in the previous 30 days, $57 \%$ reported 1 to 3 drinks per day, and $33 \%$ reported 3 or more drinks/day.

Table 3 lists the sensitivity and specificity of the $\% \mathrm{CDT}$ in the sample by group. The overall sensitivity of the \%CDT test was $61 \%$ using 90 or more drinks in 30 days as a definition of a true positive. Eleven of the 18 patients who drank 90 or more drinks (3 drinks or $42 \mathrm{~g}$ of alcohol per day) 
Table 2. Frequency of Six Categories of Alcohol Consumption and Presence of DSM-IV Criteria for Alcohol Abuse or Dependence by Diabetes and Hypertension Status $(\mathrm{N}=799)$

\begin{tabular}{|c|c|c|c|c|c|}
\hline & $\begin{array}{l}\text { Diabetic Only } \\
\quad(\mathrm{n}=89)\end{array}$ & $\begin{array}{l}\text { Hypertensive Only } \\
\qquad(\mathrm{n}=299)\end{array}$ & $\begin{array}{c}\text { Both Diabetic } \\
\text { and Hypertensive } \\
(\mathrm{n}=209)\end{array}$ & $\begin{array}{l}\text { Neither Diabetic } \\
\text { nor Hypertensive } \\
\quad(\mathrm{n}=202)\end{array}$ & $\begin{array}{l}\text { All Patients } \\
(\mathrm{n}=799)\end{array}$ \\
\hline \multicolumn{6}{|c|}{ Alcohol consumption, past 30 days* } \\
\hline 0 drinks & $50(56 \%)$ & $115(38 \%)$ & $109(52 \%)$ & $63(31 \%)$ & $337(42 \%)$ \\
\hline 1 to 29 drinks & $33(37 \%)$ & $133(44 \%)$ & $82(39 \%)$ & $112(55 \%)$ & $360(45 \%)$ \\
\hline 30 to 59 drinks & $3(3 \%)$ & $25(8 \%)$ & $12(6 \%)$ & $17(8 \%)$ & $57(7 \%)$ \\
\hline 60 to 89 drinks & $1(1 \%)$ & $17(6 \%)$ & $3(1 \%)$ & $6(3 \%)$ & $27(3 \%)$ \\
\hline 90 to 119 drinks & $0(0 \%)$ & $3(1 \%)$ & $1(0 \%)$ & $0(0 \%)$ & $4(1 \%)$ \\
\hline $120+$ drinks & $2(0.02 \%)$ & $6(2 \%)$ & $2(1 \%)$ & $4(2 \%)$ & $14(2 \%)$ \\
\hline \multicolumn{6}{|l|}{ Alcohol Abuse } \\
\hline Current & $0(0 \%)$ & $7(2 \%)$ & $6(3 \%)$ & $4(2 \%)$ & $17(2 \%)$ \\
\hline Lifetime & $25(28 \%)$ & $99(33 \%)$ & $74(35 \%)$ & $85(42 \%)$ & $283(35 \%)$ \\
\hline None & $64(82 \%)$ & $193(65 \%)$ & $119(62 \%)$ & $113(56 \%)$ & $499(63 \%)$ \\
\hline \multicolumn{6}{|c|}{ DSM-IV Alcohol Dependence } \\
\hline Current & $1(1 \%)$ & $10(3 \%)$ & $8(4 \%)$ & $4(2 \%)$ & $23(3 \%)$ \\
\hline Lifetime & $11(12 \%)$ & $45(15 \%)$ & $25(12 \%)$ & $37(18 \%)$ & $118(15 \%)$ \\
\hline None & $77(87 \%)$ & $244(82 \%)$ & $176(84 \%)$ & $161(80 \%)$ & $658(82 \%)$ \\
\hline
\end{tabular}

* 1 standard drink $=14 \mathrm{~g}$ of alcohol (12 ounces of beer, 5 ounces of wine, or 1.5 ounces of spirits). The six consumption categories are equivalent to abstinence, $<1$ drink/day, 1 to 2 drinks/day, 2 to 3 drinks/day, 3 to 4 drinks/day, and 4 or more drinks/day. These categories are used in national epidemiological studies (31).

had a \%CDT level higher than $2.5 \%$. The specificity of the \%CDT test was $85 \%$. Combining the patients who drank 90 or more drinks and the patients who met current criteria for alcohol abuse and dependence as true cases lowered the sensitivity to $40 \%$ and lowered specificity to $80 \%$.

Figure 1 illustrates the interquartile range of the \%CDT levels in the total sample. The sample is divided into 3 groups: persons who were abstinent, persons who reported 1 to 89 drinks in the previous 30 days, and persons drinking 90 or more drinks in the last 30 days. The median \%CDT level was 2.0 in the abstinent group, 2.1 in the moderate drinking group, and 2.7 in the high-risk drinking group. The \%CDT levels ranged from 0.8 to 6.4 in the abstinent group, 0.9 to 11 in the moderate group, and 1.8 to 7.4 in the high-risk drinking group.

Figure 2 presents a ROC curve for the CDT test in the population of 799 subjects. True cases were defined as persons who reported 90 or more standard drinks in the previous 30 days. Using a CDT cut-off score of 2.6 or greater provided a sensitivity of $61 \%$ and a specificity of $85 \%$. The area under the ROC curve is 0.803 . The ROC allows clinicians and health care systems to weigh the tradeoffs in terms of sensitivity and specificity when alternate cut-off values are used to indicate a positive \%CDT test. For example, if clinicians want to minimize the risk of false-positive tests, they may want to increase the CDT cutoff level to 3.0, where the falsepositive rate decreases from $15 \%$ to $3 \%$. Test sensitivity falls to $39 \%$ when using 3.0 as the cutoff value for a positive \%CDT.

Test-retest reliability was determined by inviting all subjects with an elevated CDT and those who drank 60 or more drinks in the previous 30 days to participate in repeat CDT tests 2 and 4 months after baseline. Eighty-two subjects with a

Table 3. Sensitivity and Specificity of \%CDT in a Sample of Patients with Diabetes and/or Hypertension and Control Subjects $(n=799)$

$\begin{array}{cc}\begin{array}{c}\text { Sensitivity } \\ \text { (Positive \%CDT/True Cases) }\end{array} & \begin{array}{c}\text { Specificity }^{*} \\ \text { (Negative \%CDT/True-N }\end{array} \\ 40 \%(2 / 5) & 89 \%(260 / 293) \\ 58 \%(7 / 12) & 87 \%(430 / 496) \\ 67 \%(2 / 3) & 90 \%(185 / 206) \\ 100 \%(4 / 4) & 79 \%(154 / 196) \\ 61 \%(11 / 18) & 85 \%(659 / 779)\end{array}$

Diabetic

Hypertensive

Both diabetic and hypertensive

Neither diabetic nor hypertensive

All patients in study

$$
\begin{gathered}
58 \%(7 / 12) \\
67 \%(2 / 3) \\
100 \%(4 / 4)
\end{gathered}
$$$$
>89 \text { drinks in the past } 30 \text { days. }
$$ 


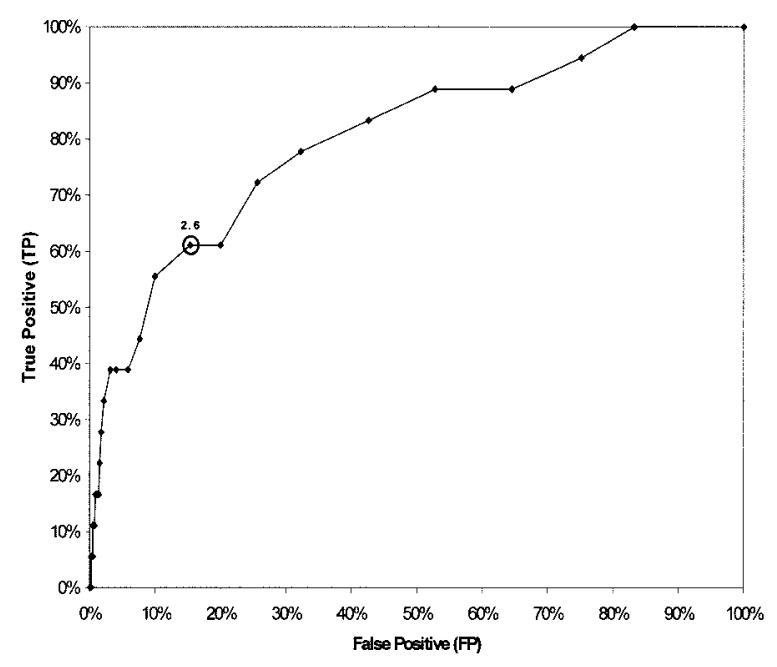

Figure 2. Receiver operator curve for \%CDT test in a sample of 799 patients with diabetes and/or hypertension and control subjects. The ROC curve plots sensitivity (true-positive) vs. 1 - specificity (false-positive) rates for the \%CDT test. A case was defined as a subject who reported 90 or more standard drinks in the previous 30 days. The specificity and sensitivity will vary depending on the \%CDT value selected. For example, if one selects the cut-off currently recommended, $2.6 \% \mathrm{CDT}$, the sensitivity is $\mathbf{6 1 \%}$ and specificity is $85 \%$. If one selects $3.0 \%$ CDT, the sensitivity decreases to $40 \%$ and the specificity increases to $97 \%$. The area under the ROC curve $=0.803$.

$\%$ CDT $>2.5$, and 26 persons drinking 60 or more drinks in the previous 30 days participated in repeat CDT testing. The test-retest reliability of the CDT test in the sample with 2- and 4-month data found a reliability coefficient $\alpha$ of 0.92 and the analysis of variance intraclass correlation $\mathrm{R}$ was 0.94. This suggests the repeat testing is stable for at least 4 months' follow-up.

\section{Discussion}

This is the first large study examining the psychometric properties of the \%CDT test in primary care patients being treated with medication for type 2 diabetes and hypertension. Prior research found that the CDT test has reasonable psychometric properties in patients being treated for alcohol dependence $^{32}$ and for screening high-risk medical and surgical patients. ${ }^{18,20}$ The study suggests CDT tests can provide useful information to primary care clinicians in their care of persons with these chronic illnesses. This study found that the \%CDT test was positive in more than $60 \%$ (ie, sensitivity) of adults drinking 90 or more drinks in the previous 30 days (42 or more grams of alcohol per day). The specificity was $85 \%$. There were minimal differences in the validity of the test by gender or group. The test-retest reliability was 0.94 .

Strengths of the study included a large sample of 799 primary care patients. The study population was randomly selected from all patients being treated for diabetes and hypertension from a diverse sample of patients receiving care at 8 primary care clinics. These clinics included urban and rural sites that are representative of primary care practices in the Midwest. The findings may be generalizable to other primary care practices in the United States with similar patient demographics. Experienced research staff conducted face-to-face confidential interviews with research subjects to minimize under-reporting of alcohol use. The \%CDT testing was conducted by an experienced laboratory scientist at the University of Wisconsin Hospital. The \%CDT test results obtained at the University of Wisconsin were very similar to those obtained on a subsample retested at 2 reference laboratories.

Limitations of the study are related to the selection process, criterion standard, and confirmation of self-report. First, there is the potential for selection bias, because this study depended on volunteer participation and subject's ability to participate in the research interviews. The project attempted to minimize selection bias by guaranteeing patient confidentiality, providing financial incentives, and using experienced research staff. The selection of a "criterion standard" for alcohol biomarkers continues to depend primarily on patient self-report. The study used the current state-of-the-art method of collecting alcohol use information, namely the 30day timeline follow-back calendar method. Traditional alcohol biomarkers such as MCV and GGT levels have been shown to be less sensitive than self-report and are not useful as criterion standards.

Other potential methods of confirming patient self-report include medical record reviews and family member collaboration. For this study, we conducted medical record reviews on all 799 patients. We discovered no alcohol use not reported by the patient, and more than half of the medical records of heavy-drinking research subjects revealed no documentation of the patient's alcohol use. Prior 
studies conducted by the authors found that family members interviewed to corroborate patient selfreport reported less alcohol use than provided by the research subject. ${ }^{33}$ These observations confirm that patient self-report, using standard research methods, is the most valid "criterion standard" in a primary care sample.

The sensitivity of the \%CDT test has been reported to vary from $20 \%$ to $95 \%$ depending on the population of interest. Studies testing the \%CDT in samples of persons recruited from alcohol treatment programs found the highest sensitivity rates of $80 \%$ to $95 \%,{ }^{34,35}$ whereas those studies that identified subjects in general medical settings reported the lowest sensitivity rate of $15 \%$ to $60 \% .^{36,37}$ Studies in high-risk trauma patients and surgical patients found sensitivities in the range of $40 \%$ to $80 \% .{ }^{18,20}$ As with most screening tests, the accuracy of the \%CDT test varies depending on the prevalence of heavy alcohol use in the population being studied. The sensitivity reported in our sample $(61 \%)$ is higher than reported in previous general clinical settings.

The specificity of the \% CDT test also varies by population and clinical setting. Although initial studies suggested \%CDT tests were highly specific for high-risk alcohol use, ${ }^{38}$ newer studies suggest a more complicated picture. Biological factors that may increase \%CDT levels are female hormones, iron stores, medication, end-stage liver disease, genetic variants, low body mass index, catabolic states, and chronic pulmonary disease. ${ }^{24-28}$ Common mechanisms that have been proposed include a redirection of glycan synthesis to the sialylated forms of transferrin and increased enzymatic removal of carbohydrate chains from circulating transferrin. ${ }^{39,40}$

In this study, we found a specificity of $85 \%$. The 120 persons who were classified as false-positives were $62 \%$ women, and the mean age of this falsepositive group was similar to that of the total sample (48.8 versus 49.7 , respectively) and were distributed proportionally across the 4 groups. A number of potential explanations exist for false-positive \%CDT tests in this sample. First, some of these patients may have been under-reporting their alcohol use. Second, unknown factors, such as medication interaction, may affect \%CDT levels. Third, metabolic factors related to diabetes, lipid disorders, hypertension, and other chronic illnesses may affect \%CDT levels. This is the first study of the validity of \%CDT levels a large sample of patients with chronic medical problems. Additional research in primary care samples is needed to assess the issue of false positive \%CDT levels.

The clinical utility of the \%CDT test in primary care is not known. However, until additional studies are conducted, this study provides sufficient evidence for primary care physicians to begin to use this test in conjunction with other methods, such as questionnaires and other alcohol biomarkers such as blood alcohol levels, MCV and GGT in highrisk patients. Because the predictive value of the $\%$ CDT test seems to be marginal in populations with a low prevalence of heavy alcohol use, primary care providers may want to limit the use of \%CDT to diabetic or hypertensive patients who are not under good control. As with other screening tests, such as mammography, prostate screening, and cholesterol, careful evaluation and follow-up is necessary for patients with an elevated \%CDT test.

This study could have important implications for primary care physician treatment and follow-up strategies for patients with hypertension and/or diabetes. In this study, 9\% (29 of 298) of the diabetics and $15 \%$ of the hypertensive patients were found to be drinking 30 or more drinks in the previous 30 days or met criteria for alcohol abuse/dependence. None of these patients should drink more than 1 drink per day because of the adverse affect of alcohol on glucose and blood pressure regulation and other adverse health effects. ${ }^{41}$ There are also interactions between alcohol and some medications used to treat hypertension and diabetes. ${ }^{7}$

Our study found that fewer than $30 \%$ of the subjects who reported 30 or more drinks in the previous 30 days had alcohol use information in the medical record. This finding suggests primary care physicians may want to used the \%CDT test, in conjunction with questionnaires, to help them identify patients using alcohol above recommended levels. Identification could be followed up with brief intervention and motivational interviewing, in that prior studies suggest brief physician advice can reduce alcohol use and alcohol-related morbidity and costs. ${ }^{41}$

Assuming there are 15 million patients with diabetes $^{42}$ and 50 million patients with hyperten$\operatorname{sion}^{43}$ in the United States, the findings of our study suggest there are 1.35 million patients with diabetes and 7.5 million patients with hypertension who drink too much (28 or more drinks per 
month), who could benefit from brief physician advice. In addition to identifying high-risk drinkers, CDT levels can be used to monitor relapse. Studies have found that CDT seems to act like a glycosylated hemoglobin test. It generally decreases within 2 weeks of abstinence and will increase if persons resume drinking. ${ }^{13,32,44}$ Quarterly testing of CDT levels in unstable diabetics or hypertensive patients may be indicated to monitor reductions or increases in drinking. CDT is the only alcohol biomarker sensitive enough to monitor reductions in alcohol use or relapse.

\section{References}

1. Wei M, Gibbons LW, Mitchell TL, Kanpert JB, Blair SN. Alcohol intake and incidence of type 2 diabetes in men. Diabetes Care 2000;23:18-22.

2. Tsumura K, Hayashi T, Suematsu C, Endo G, Fujii S, Okada K. Daily alcohol consumption and the risk of type 2 diabetes in Japanese men: The Osaka Health Survey. Diabetes Care 1999;22:1432-7.

3. Turner BC, Jenkins E, Kerr D, Sherwin RS, Cavan DA. The effect of evening alcohol consumption on next-morning glucose control in type 1 diabetes. Diabetes Care 2001;24:1888-93.

4. Kao WH, Puddey IB, Boland LL, Watson RL, Brancati FL. Alcohol consumption and the risk of type 2 diabetes mellitus: atherosclerosis risk in communities study. Am J Epidemiol 2001;154:748-57.

5. Wartenberg AA. Management of common medical problems. In: Graham AW, Schultz TK, Wilford BB, editors. Principles of addiction medicine. Chevy Chase (MD): American Society of Addiction Medicine; 1998. p. 731-9.

6. Sikkink J, Fleming M. Adverse health effects and medical complications of alcohol, nicotine and drug use. In: Fleming MF, Barry KL, editors. Addictive disorders. St. Louis: Mosby Year Book; 1992. p. $145-68$.

7. Weathermon R. Alcohol and medication interactions. Alcohol Health Res World 1999;23:40-54.

8. Fleming MF, Mihic SJ, Harris RA. Ethanol. In: Goodman \& Gilman's the pharmacological basis of therapeutics. 10th ed. New York: McGraw-Hill Medical Pub; 2001. p. 429-46.

9. Godsland IF, Leyva F, Walton C, Worthington C, Stevenson JC. Association of smoking and physical activity with risk factors for coronary heart disease and diabetes in the first follow-up cohort of the Heart Disease and Diabetes Risk Indicators in a Screened Cohort study (HDDDRISC-1). J Intern Med 1998;244:33-41.

10. Dawson DA. US low-risk drinking guidelines: an examination of four alternatives. Alcohol Clin Exp Res 2000;24:1820-9.

11. Physicians' guide to helping patients with alcohol problems. Bethesda (MD): US Department of Health and Human Services, Public Health Service, National Institutes of Health. NIH publication no. 95-3769. Available at: http://www.niaaa.nih.gov/ publications/physicn.htm

12. Harasymiw J, Bean P. The combined use of the early detection of alcohol consumption (EDAC) test and carbohydrate-deficient transferrin to identify heavy drinking behaviour in males. Alcohol Alcohol 2001; 36:349-53.

13. Montalto NJ, Bean P. Use of contemporary biomarkers in the detection of chronic alcohol use. Med Sci Monit 2003;9:RA285-90.

14. Stowell L, Stowell A, Garrett N, Robinson G. Comparison of serum $\beta$-hexosaminidase isoenzyme $\beta$ activity with serum carbohydrate-deficient transferrin and other markers of alcohol abuse. Alcohol Alcohol 1997;32:703-14.

15. Center for Medicare \& Medicaid Services, (CMS), HHS. Medicare program; negotiated rulemaking: coverage and administrative policies for clinical diagnostic laboratory services. Final rule. Fed Regist 2001;66:58788-890.

16. Bird WF Jr. Detecting alcohol abuse: the value of carbohydrate-deficient transferrin. J Insur Med 1997;29:126-35.

17. Sillanaukee P, Aalto M, Seppa K. Carbohydratedeficient transferrin and conventional alcohol markers as indicators for brief intervention among heavy drinkers in primary health care. Alcohol Clin Exp Res 1998;22:892-6.

18. Tonnesen H, Carstensen M, Maina P. Is carbohydrate-deficient transferrin a useful marker of harmful alcohol intake among surgical patients?. Eur J Surg 1999;165:522-7.

19. Gomez A, Conde A, Aguiar JA, Santana JM, Jorrin A, Betancor P. Diagnostic usefulness of carbohydrate-deficient transferrin for detecting alcoholrelated problems in hospitalized patients. Alcohol Alcohol 2001;36:266-70.

20. Spies CD, Kissner M, Neumann T, Blum S, Voigt $\mathrm{D}$, Funk T, et al. Elevated carbohydrate-deficient transferring predicts prolonged intensive care unity stay in traumatized men. Alcohol Alcohol 1998;33: 661-9.

21. Wetterling T, Kanitz RD, Renner F, Fischer D. Does CDT predict the severity of alcohol withdrawal syndrome? Alcohol Clin Exp Res 1998;22: 1053-6.

22. Mundle G, Ackerman K, Munkes J, Steinle D, Mann $\mathrm{K}$. Influence of age, alcohol consumption and abstinence on the sensitivity of carbohydratedeficient transferrin, gamma-glutamyltransferase and mean corpuscular volume. Alcohol Alcohol 1999;34:760-6.

23. Brathen G, Bjerve KS, Brodtkorb E, Helde G, Bovim G. Detection of alcohol abuse in neurological patients: variables of clinical relevance to the accu- 
racy of the \%CDT-TIA and CDTect methods. Alcohol Clin Exp Res 2001;25:46-53.

24. Sillanaukee P, Alho H, Strid N, et al. Effect of hormone balance on carbohydrate-deficient transferrin and gamma-glutamyltransferase in female social drinkers. Alcohol Clin Exp Res 2000;24:1505-9.

25. Whitfield JB, Zhu G, Heath AC, Powell LW, Martin NG. Effects of alcohol consumption on indices of iron stores and or iron stores on alcohol intake markers. Alcohol Clin Exp Res 2001;25:1037-45.

26. Whitfield JB, Fletcher LM, Murphy TL, et al. Smoking, obesity, and hypertension alter the doseresponse curve and test sensitivity of carbohydratedeficient transferrin as a marker of alcohol intake. Clin Chem 1998;44:2480-9.

27. Meerkerk GJ, Njoo KH, Bongers IM, Trienekens P, vanOers JAM. Comparing the diagnostic accuracy of carbohydrate-deficient transferrin, gamma-glutamyltransferase, and mean cell volume in a general practice population. Alcohol Clin Exp Res 1999;23: 1052-9.

28. Sillanaukee P, Strid N, Jousilahti P, et al. Association of self-reported diseases and health care use with commonly used laboratory markers for alcohol consumption. Alcohol Alcohol 2001;36:339-45.

29. Bean P. Carbohydrate-deficient transferrin in the assessment of harmful alcohol consumption: diagnostic performance and clinical significance. Addiction Biology 1999;4:151-61.

30. Sobell LC, Brown J, Leo GI, Sobell MB. The reliability of the Alcohol Timeline Followback when administered by telephone and by computer. Drug Alcohol Depend 1996;42:49-54.

31. Grant BF. Prevalence of the proposed DSM-IV alcohol use disorders: United States, 1988. Br J Addict 1992;87:309-16.

32. Anton R, Lieber C, Tabakoff B, CDTect Study Group. Carbohydrate-deficient transferrin and gamma-Glutamyltransferase for the detection and monitoring of alcohol use: results from a multisite study. Alcohol Clin Exp Res 2002;26:1215-22.

33. Fleming MF, Barry KL, Manwell LB, Johnson K, London R. Brief physician advice for problem alcohol drinkers: a randomized controlled trial in community-based primary care practices. JAMA 1997;277:1039-45.

34. Allen JP, Sillamaukee P, Anton R. Contribution of carbohydrate-deficient transferrin to gamma glu- tamyl transpeptidase in evaluating progress of patients in treatment for alcoholism. Alcohol Clin Exp Res 1999;23:115-20.

35. Anton R, Moak D, Latham P. Carbohydratedeficient transferrin as an indicator of drinking status during a treatment outcome study. Alcohol Clin Exp Res 1996;20:841-6.

36. Aertgeerts B, Buntinx F, Ansoms S, Fevery J. Screening properties of questionnaires and laboratory tests for the detection of alcohol abuse or dependence in a general practice population. Br J Gen Pract 2001; 51:206-17.

37. Huseby N, Nillsen O, Erfurth A, Wetterling T, Kanitz R. Carbohydrate-deficient transferrin and alcohol dependency: variation in response to alcohol intake among different groups of patients. Alcohol Clin Exp Res 1997;21:201-5.

38. Stibler H, Borg S, Allgulander C. Clinical significance of abnormal heterogeneity of transferrin in relation to alcohol consumption. Acta Med Scand 1979;206:275-81.

39. Brathen G, Bjerve KS, Brodtkorb E, Bovim G. Validity of carbohydrate-deficient transferrin and other markers as diagnostic aids in the detection of alcohol related seizures. J Neurol Neurosurg Psychiatry 2000;68:342-8.

40. de Jong G, van Noort WL, Feelders RA, de JeuJaspars CMH, van Eijk HG. Adaptation of transferrin protein and glycan synthesis. Clin Chim Acta 1992;212:27-45.

41. Fleming MF, Mundt MP, French MT, Manwell LB, Stauffacher EA, Barry KL. Brief physician advice for problem drinkers: long-term efficacy and benefitcost analysis. Alcohol Clin Exp Res 2002;26:36-43.

42. Centers for Disease Control and Prevention. National diabetes fact sheet: general information and national estimates on diabetes in the United States, 2000. Atlanta (GA): US Department of Health and Human Services, Centers for Disease Control and Prevention; 2002.

43. American Heart Association. 2002 Heart and Stroke Statistical Update. Dallas (TX): American Heart Association; 2001.

44. Heinemann A, Sterneck M, Kuhlencordt R, et al. Carbohydrate deficient transferrin: diagnostic efficiency among patients with end-stage liver disease before and after liver transplantation. Alcohol Clin Exp Res 1998;22:1806-12. 